\title{
Social rank and rejection sensitivity as mediators of the relationship between insecure
}

\author{
attachment and disordered eating
}

Tara De Paoli ${ }^{\mathrm{a}}$, Matthew Fuller-Tyszkiewicz ${ }^{\mathrm{b}, \mathrm{c}}$, Emma Halliwell $^{\mathrm{d}}$, Francis Puccio ${ }^{\mathrm{a}}$, \& Isabel $\operatorname{Krug}^{\mathrm{a} *}$

${ }^{\text {a }}$ Melbourne School of Psychological Sciences, The University of Melbourne, Melbourne, VIC, Australia.

${ }^{\mathrm{b}}$ School of Psychology, Deakin University, Geelong, VIC, Australia.

${ }^{\mathrm{c}}$ Centre for Social and Early Emotional Development, Deakin University, Burwood, VIC, Australia.

${ }^{\mathrm{d}}$ Centre for Appearance Research, The University of the West of England, Bristol, United Kingdom.

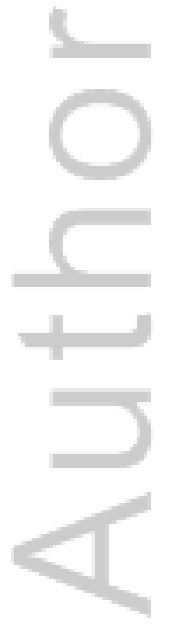

This is the author manuscript accepted for publication and has undergone full peer review but has not been through the copyediting, typesetting, pagination and proofreading process, which may lead to differences between this version and the Version of Record. Please cite this article as doi: $10.1002 /$ erv.2537

This article is protected by copyright. All rights reserved. 
*Address for correspondence:

Isabel Krug, PhD, Senior Lecturer in Clinical Psychology, University of Melbourne Psychology Clinic, 14-20 Blackwood Street, North Melbourne VIC 3051, Australia; Tel: + 61390358551; Fax: +61393264774; E-mail: isabel.krug@unimelb.edu.au

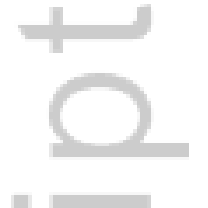

1

\section{Abstract}

Aim: The current study assessed a new interpersonal model for eating disorders (EDs), in which interpersonal rejection sensitivity (RS), appearance-based RS, and social rank were hypothesised to mediate the relationship between insecure attachment and disordered eating. Method: The sample comprised a clinical ED group $(\mathrm{N}=122)$ and a control group $(\mathrm{N}=622)$. Participants were asked to complete a number of self-report measures related to the variables of interest. Results: Invariance testing indicated that the model was structurally invariant (different across groups). For the ED group, appearance-based RS and social rank were significant mediators of the relationship between insecure attachment and disordered eating. For the controls, the relationship between insecure attachment and disordered eating was mediated through multiple pathways involving interpersonal RS, appearance-based RS and social rank. Conclusion: These findings may inform existing therapies such as interpersonal psychotherapy for EDs, by emphasising the role of sensitivity to rejection in the development and maintenance of disordered eating.

Keywords: eating disorder, disordered eating, social rank, rejection sensitivity, appearance 


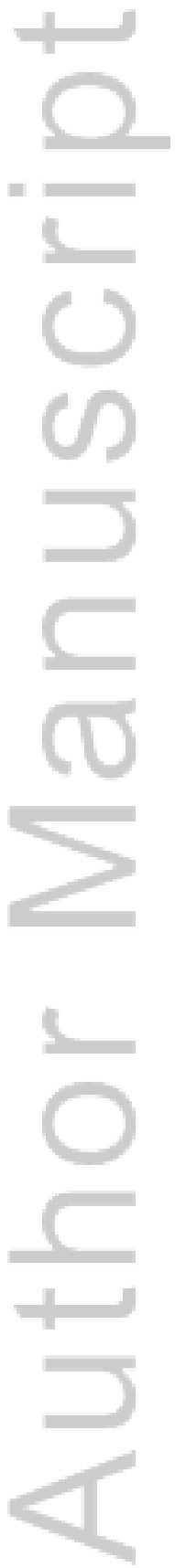

This article is protected by copyright. All rights reserved. 


\section{Social rank and rejection sensitivity as mediators of the relationship between insecure attachment and disordered eating}

Insecure attachment styles are commonly reported in disordered eating populations (CaglarNazali et al., 2014; Illing, Tasca, Balfour, \& Bissada, 2010; Ward, Ramsay, \& Treasure, 2000), and it is estimated that approximately 96 to 100 percent of women with eating disorders (EDs) have an insecure attachment style (Dakanalis et al., 2014). Several existing interpersonal models of EDs including the interpersonal functioning maintenance model (Arcelus, Haslam, Farrow, \& Meyer, 2013), the eating disorder-specific model of interpersonal psychotherapy (IPT-ED; Rieger et al., 2010), and the cognitive-interpersonal maintenance model of anorexia nervosa (Schmidt \& Treasure, 2006; Treasure \& Schmidt, 2013), have attempted to articulate the role of attachment in the development and maintenance of eating pathology. These models propose that insecure attachment is present before ED onset (Treasure \& Schmidt, 2013), and may lead to a fear of negative social evaluation (Arcelus et al., 2013), which then becomes central in triggering ED symptoms such as restriction or binge eating, and inevitably serves to maintain the disorder (Arcelus et al., 2013; Rieger et al., 2010). While there is some support for these propositions (e.g., Goddard et al., 2013), the majority of the proposed pathways have yet to be empirically evaluated. Moreover, other constructs related to negative evaluation that may also account for the link between insecure attachment and EDs, such as social rank (Connan, Troop, Landau, Campbell, \& Treasure, 2007) and rejection sensitivity (RS) (Cardi, Di Matteo, Corfield, \& Treasure, 2013), have not yet been incorporated into these models. Therefore, the current 
study tests an expanded form of the IPT-ED model, by incorporating these additional paths, and testing a whole model rather than individual components in isolation. The model was tested across clinical ED and control samples to evaluate generalisability.

Attachment is gradually shaped by early experiences with attachment figures, and eventually results in a fairly stable attachment style or orientation, which can be conceptualised as either secure or insecure (Bowlby, 1973; Fraley \& Shaver, 2000; Main, Kaplan, \& Cassidy, 1985). Attachment style influences how people perceive and react during various types of social interactions (Vrtička \& Vuilleumier, 2012). Individuals with insecure attachment styles may rely heavily on relationships to validate their self-worth (Bartholomew \& Horowitz, 1991). Alternatively, they may be socially avoidant, and steer away from close involvement with others to protect themselves against anticipated rejection (Bartholomew \& Horowitz, 1991).

Insecure attachment may foster general concerns about negative social evaluation, defined by Rieger et al. (2010) as actual or perceived negative feedback regarding one's value to another individual or group. This negative social evaluation may include a sensitivity to rejection and the perception that one is of low social rank. RS is considered an individual difference in the tendency to anxiously expect, readily perceive, and overreact to real or imagined rejection (Downey \& Feldman, 1996), while low social rank is associated with the perception of being inferior to others, less attractive, and an outsider (Gilbert, 1992). Within the context of ED symptoms, sensitivity to rejection may make individuals particularly vulnerable to negative comments about their appearance and/or motivate them to strive for the perceived ideal in order to avoid such criticism. Likewise, a general belief that one is of low 
social rank may generalise to beliefs about one's appearance, such that the individual perceives their appearance does not meet an objective standard, and thus they are appearance dissatisfied and strive to attain the ideal (Pinto-Gouveia, Ferreira, \& Duarte, 2015). In turn, these body image concerns may prompt ED behaviours.

Accumulated literature provides support for both RS and low social rank in the interpersonal difficulties experienced by individuals with EDs. RS is associated with the learnt expectation to selectively attend to hostile social cues, and the tendency to misinterpret ambiguous social cues as rejection (Downey \& Feldman, 1996). Cardi et al. (2013) found that lifetime ED patients showed an attentional bias to rejecting faces and difficulty disengaging attention from these stimuli. Further, this attentional bias to rejection was correlated with adverse childhood experiences. This altered attention pattern for social stimuli has also been found in neuropsychological studies of ED populations, and is thought to involve overlapping social cognition and reward systems, which lead to a disruption of adaptive responses in social processing (Via et al., 2015).

While general RS may be expected to have negative outcomes, it is likely that RS specifically related to appearance is most closely aligned with ED outcomes (Rieger et al., 2010). Appearance-based RS is regarded as a dispositional personality processing system characterised by anxious concerns and expectations about being rejected specifically based on one's physical appearance (Park, 2007). Individuals with appearance-based RS associate physical flaws with rejection, resulting in preoccupation with their bodies and motivation to engage in behaviours to appear attractive to avoid rejection by others (Park, 2007). Appearance-based RS has been found to predict disordered eating in community samples 
(Park, 2007) and the tendency to make social comparisons based on appearance (Calogero, Park, Rahemtulla, \& Williams, 2010).

In terms of social rank, ED patients commonly report features consistent with low social rank including an increased tendency for submission and the perception of being inferior to others (Connan et al., 2007; Troop, Allan, Treasure, \& Katzman; 2003). These behaviours are also evident to a lesser extent in recovered ED patients, suggesting that it may be a trait vulnerability factor (Cardi, Di Matteo, Gilbert, \& Treasure, 2014; Connan et al., 2007). Low social rank has also been shown to affect attentional processing in ED patients. Cardi et al. (2014) investigated the automatic processing of social rank-related stimuli in ED patients, recovered ED patients, and controls, and found that ED patients showed heightened sensitivity toward social rank-related stimuli compared to controls. Conversely, recovered ED patients showed an intermediate profile between currently ill ED patients and controls. Selfreport data in the same study confirmed the behavioural findings, indicating that ED patients viewed themselves to be lower in social rank, and had higher submissive behaviours and internal and external shame relative to controls. In a more recent study, Troop (2016) found that life events associated with a loss of social status (e.g., decreased reputation) were related to eating pathology, but only in women who perceived themselves to be low in social rank. Taken together, accumulated findings suggest that, at the level of diagnosis, ED individuals are more likely to show insecure attachment styles, and exhibit heightened RS and perceptions of low social rank. Insecure attachment has also been tied to specific ED symptoms (Illing et al., 2010), suggesting that there may be a direct, causal relationship between insecure attachment and ED symptom development and maintenance, rather than 
simply a non-causal co-morbidity. Nevertheless, despite the proliferation of interpersonal models that emphasise the role of insecure attachment in the development of EDs, there have been few investigations into potential mediating social factors of this relationship. To date, there are no known studies that have tested Arcelus et al.'s (2013) or Rieger et al.'s (2010) interpersonal models, while existing studies (Goddard et al., 2011; 2013) of the cognitive interpersonal maintenance model (Schmidt \& Treasure, 2006) have only focused on specific components of the model. Moreover, while the pattern of findings for interpersonal maintaining factors are encouraging, there have been no known attempts to test the role of attachment in the aforementioned models. Thus, the current study expands extant interpersonal models and addresses gaps within the literature by examining the variables of attachment, interpersonal RS, appearance-based RS, social rank, and disordered eating concurrently. To do this, a new interpersonal model for EDs (outlined in Figure 1) was tested in which interpersonal RS, appearance-based RS, and social rank, were hypothesised to mediate the relationship between insecure attachment and disordered eating. The study utilised a clinical ED sample and a control sample to determine whether the relationships in the model differed across the two groups.

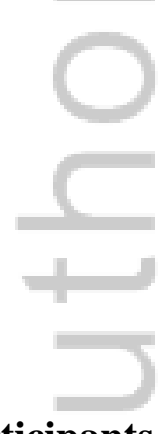
Insert Figure 1 about here

\section{METHOD}

\section{Participants}


ED participants were ascertained from different ED clinics and services across Australia. The sample consisted of 122 participants with a lifetime ED diagnosis $(98 \%$ female, $M$ age $=$ 25.16 years, $S D=7.60)$ including 56 anorexia nervosa restricting $(\mathrm{AN}-\mathrm{R}), 17$ anorexia nervosa binge-purge (AN-BP), 17 bulimia nervosa (BN), 22 other specified feeding and eating disorder (OSFED/EDNOS), 10 binge eating disorder (BED). Formal ED diagnosis was determined from psychiatrist report in tertiary settings according to the Diagnostic and Statistical Manual of Mental Disorders (DSM-5; American Psychiatric Association, 2013) criteria, and by self-report in other settings. Participants varied in treatment stage, and 40 (32.79\%) participants reported that they were currently recovered from their ED. Average age of onset for the ED group was 15.23 years $(S D=4.32)$. Average body mass index (BMI) was $20.98(S D=4.59)$ (healthy) and 18 of $122(14.75 \%)$ participants reported a BMI over 25 (overweight/obese).

A comparison group was recruited from the community and a first year psychology university course. Control participants were screened for lifetime ED diagnosis and disordered eating behaviours using the Eating Disorders Examination Questionnaire (EDE-Q; clinical cut-off $>4$; Fairburn \& Beglin, 1994) and 45 participants were removed. A total of 622 control participants $(79 \%$ female, $M$ age $=22.01$ years, $S D=8.63)$ were included in the final analyses. Average BMI was $22.35(S D=4.70)$ (healthy) and 97 of $622(15.59 \%)$ participants reported a BMI over 25 (overweight/obese). Ethical approval was obtained from a university in Melbourne and the hospitals from where the clinical ED sample was recruited.

\section{Measures}

This article is protected by copyright. All rights reserved. 
Sociodemographics and clinical information. Information on participant age, height, weight, ethnicity, employment status, marital status, highest completed education, lifetime ED status, and age of ED onset were obtained. BMI was calculated as the ratio of weight $(\mathrm{kg})$ to height squared $\left(\mathrm{m}^{2}\right)$.

Disordered eating. The Eating Disorder Inventory 3 (EDI-3; Garner, 2004) was used to assess disordered eating. The EDI-3 is a 91-item self-report measure that assesses symptomatology associated with EDs. The items are scored on a 6-point rating scale, which is then scored using a 0-4 system. For the current study the Drive for Thinness, Body Dissatisfaction, and Bulimia subscales of the Eating Disorder Risk Composite were used. Cronbach's alpha for the current study was drive for thinness .91, body dissatisfaction .91 and bulimia .88 .

Attachment style. Attachment style was assessed using the Revised Experiences in Close Relationships scale (ECR-R; Fraley, Waller, \& Brennan, 2000). The ECR-R is based on a dimensional model of attachment and is a 36-item self-report measure that assesses attachment anxiety and attachment avoidance in current relationships. The ECR-R is often used as an indicator of attachment across the lifespan due to anticipated attachment stability formed from early experiences (Hazan \& Shaver, 1987). The items are scored on a 5-point rating scale from 1 (strongly disagree) to 7 (strongly agree). Cronbach's alpha in the current study was attachment anxiety .94 and attachment avoidance .94 .

Interpersonal RS. Interpersonal RS was measured using the Rejection Sensitivity Questionnaire (RSQ; Downey \& Feldman, 1996). The RSQ is an 18-item self-report measure that presents brief scenarios to assess RS along two dimensions: rejection concern (extent to 
which the participant would be concerned or anxious about a significant other's response to an important request) and acceptance expectancy (extent to which the participant would expect a significant other to honour their request). These dimensions are measured on separate scales. For each situation, respondents are asked to indicate: rejection concern 1 (very unconcerned) to 6 (very concerned) and their acceptance expectancy 1 (very unlikely) to 6 (very likely). In accordance with the test authors' adoption of an expectancy-value model, RS was calculated by weighting the expected likelihood of rejection by the degree of concern over its occurrence. Specifically, expectancy of acceptance was reversed scored to index expectancy of rejection (expectancy of rejection $=7$ - expectancy of acceptance). Total RS score was then calculated as the product of rejection concern and expectancy of rejection. The Cronbach's alpha for the overall RS scores in the current study was .85.

Appearance-based RS. Appearance-based RS was measured using the AppearanceBased RS Scale (Appearance-RS Scale; Park, 2007). The Appearance-RS Scale is an 18-item self-report measure that presents brief scenarios to assess rejection based on appearance. Similar to the RSQ, the Appearance-RS Scale assesses rejection along the two dimensions of rejection concern and rejection expectancy. For each situation, respondents are asked to indicate: rejection concern 1 (very unconcerned) to 6 (very concerned) and their rejection expectancy 1 (very unlikely) to 6 (very likely). Overall appearance-based RS score was calculated by multiplying the degree of rejection concern with the degree of rejection expectancy. Cronbach's alpha for the overall RS scores in the current study was .96.

Social rank. Self-perceived social rank was measured using the Social Comparison Rating Scale (SCRS; Allan \& Gilbert, 1995). The SCRS is an 11-item self-report measure 
that uses a semantic differential methodology to measure a respondent's judgment of themselves in relation to others. The respondent is asked to rate from 1 to 10 on a series of bipolar constructs "In relation to others I feel..." 1 (inferior) to 10 (superior), 1 (an outsider) to 10 (an insider), 1 (unattractive) to 10 (more attractive), etc. The SCRS is divided into three subscales social comparison of rank, social comparison of group fit, and social comparison of attractiveness. The items are added to derive a total score with low scores indicating low rank self-perceptions. Cronbach's alpha for the total score in the current study was .95 .

\section{Procedure}

Consenting adults were given access to an online questionnaire via Qualtrics Online Survey Software. All participants read a consent form informing them of the voluntary nature of the study, before proceeding with the questionnaire. The participants were then asked to complete the sociodemographic questions and the self-report measures related to the variables of interest. Participants were informed that they could withdraw from the study at any time.

\section{Statistical Analyses}

All descriptive, correlational, and group-difference based analyses were conducted using IBM SPSS 24.0. Between-group analyses of the sociodemographic factors were conducted using chi-square and $t$-tests to identify differences between the clinical ED sample and the control sample. Effect size was determined by Cohen's $d$ with $d=0.2$ indicating a small effect, $d=$ 0.5 medium effect, and $d=0.8$ indicating a large effect (Cohen, 1992). Two-tailed bivariate correlations were conducted separately for the clinical ED group and the control group. 
Correlations were derived between attachment, interpersonal RS, appearance-based RS, social rank, and disordered eating. Strength of the correlation was determined by Pearson's r, with $.10<\mathrm{r}<.30$ indicating weak correlations, $.30<\mathrm{r}<.50$ medium correlations, and $\mathrm{r}>.50$ indicating a strong relationship (Cohen, 1992).

Path analysis using MPlus software was conducted to test the hypothesised model in Figure 1. Structural invariance testing was undertaken to investigate whether the relationships in the model were equivalent across the ED group and the control group. The model was controlled for age and gender due to the majority of the ED sample being female. Bootstrapping (5000 bootstraps) was used to assess mediation (Shrout \& Bolger, 2002). Model modification was completed where improvements to the fit of the data could be obtained and if theoretically justifiable. Given that the $\chi^{2}$ statistic is highly sensitive to large sample sizes (Tabachnick \& Fidell, 2007), adequacy of model fit was also assessed via the comparative fit index (CFI), root mean square error of approximation (RMSEA) and standardised root-mean square residual (SRMR). Following recommended criteria, CFI values above .95, RMSEA below .10 and SRMR below .08 were used to indicate acceptable fit to the data (SchermellehEngel, Moosbrugger \& Muller, 2003). The model was first tested for the ED group and the control group separately where all parameters were allowed to vary across groups (unconstrained model). Next, the model was constrained, whereby regression paths for each group were constrained to be equal and compared with the unconstrained model. A model was deemed structurally invariant (i.e., equivalent across groups), if $\triangle \mathrm{CFI}$ was less than .01 (Cheung \& Rensvold, 2002). 


\section{RESULTS}

\section{Sociodemographics}

The sociodemographic variables for the overall sample, ED group, and control group are presented in Table 1. Significant group differences were observed for age, BMI, ethnicity, education, marital status, and employment. The clinical ED participants were significantly older, had a lower BMI, and were more commonly Caucasian, married and unemployed than the controls. Conversely, the control participants were significantly younger, had a higher BMI, and were more commonly Asian, single and current students.

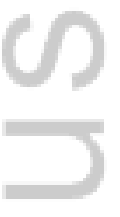

Insert Table 1 about here

\section{Differences in the variables of interest across groups}

Table 2 outlines the group differences for the means for each of the variables of interest. Across all the variables, the clinical ED participants reported significantly higher levels of insecure attachment (both anxious and avoidant), RS (both interpersonal and appearancebased), disordered eating (drive for thinness, body dissatisfaction, and bulimia) and lower social rank than the control group.

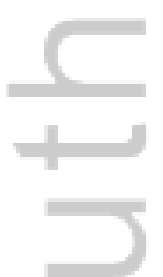

Insert Table 2 about here

\section{Correlations among study variables}

Correlations for all variables are presented separately for the ED sample and the controls in 
Table 3. For both the clinical ED group and the control group, attachment anxiety and attachment avoidance showed moderate significant positive relationships with the disordered eating variables. Interpersonal RS showed large positive significant associations with the disordered eating variables for the clinical ED group, while the control group showed moderate significant positive associations. Appearance-based RS showed large positive significant correlations with the disordered eating variables for both the clinical ED group and the control group. Finally, social rank showed medium to large negative relationships with disordered eating in the clinical ED group, and medium inverse associations in the control group.

-------- Insert Table 3 about here

\section{Invariance testing}

Invariance testing between the clinical ED group and the control group indicated that the model was structurally variant (i.e. different between groups). The fit for the unconstrained model was $\chi^{2}=31.989, \mathrm{RMSEA}=0.067, \mathrm{SRMR}=0.025, \mathrm{CFI}=0.991$, and the fit for the constrained model was $\chi^{2}=120.591, \mathrm{RMSEA}=0.065, \mathrm{SRMR}=0.070, \mathrm{CFI}=0.968$, indicating a worsening of fit $(\triangle \mathrm{CFI}=.023)$. Exploration of the modification indices suggested a large number of pathways that would need to be freed across the groups to loosen the invariance assumption, indicating that the relationships in the model differed significantly across the groups. Path analyses for each group are reported separately below. 


\section{Path analyses for the ED group}

For the clinical ED group, significant direct effects were observed for: 1.) appearance-based RS and low social rank associated with drive for thinness, 2.) attachment avoidance, appearance-based RS, and low social rank associated with body dissatisfaction, and 3.) appearance-based RS associated with bulimia (see Table 4). Significant indirect effects were found for: 1.) anxious attachment on drive for thinness through appearance-based RS, 2.) avoidant attachment on drive for thinness through social rank 3.) anxious attachment on body dissatisfaction through appearance-based RS (see Table 5). The model accounted for $43.9 \%$ of the variance for drive for thinness $\left(\mathrm{R}^{2}=0.439\right), 56.7 \%$ of the variance for body dissatisfaction $\left(\mathrm{R}^{2}=0.567\right)$, and $19.8 \%$ of the variance for bulimia $\left(\mathrm{R}^{2}=.198\right)$ for the $\mathrm{ED}$ group.

\section{Path analysis for the control group}

For the control group, significant direct effects were observed for: 1.) appearance-based RS associated with drive for thinness 2.) appearance-based RS and low social rank associated with body dissatisfaction, and 3.) avoidant attachment and appearance-based RS associated with bulimia (see Table 4). Significant indirect effects were found for: 1.) anxious attachment on drive for thinness through appearance-based RS, 2.) anxious attachment on bulimia through appearance-based RS, 3.) anxious attachment on body dissatisfaction through multiple pathways involving interpersonal RS, appearance-based RS and social rank, and 4.) avoidant attachment to body dissatisfaction through interpersonal RS and social rank (see Table 5). The model accounted for $31.6 \%$ of the variance for drive for thinness $\left(\mathrm{R}^{2}=0.316\right)$, 
$38.6 \%$ of the variance for body dissatisfaction $\left(\mathrm{R}^{2}=0.386\right)$, and $23.8 \%$ of the variance for bulimia $\left(\mathrm{R}^{2}=0.238\right)$ for the control group.

-------- Insert Table 4 and 5 about here -------

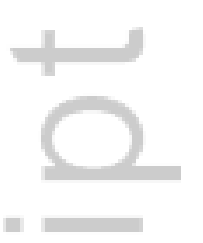

The current study represents the first concurrent investigation of attachment, interpersonal RS, appearance-based RS, and social rank within a disordered eating context. The study examined a new interpersonal model for eating pathology whereby interpersonal RS, appearance-based RS, and social rank were hypothesised to mediate the relationship between attachment and disordered eating. The model was compared across a clinical ED group and a control group, and identified several points of differences across groups in how these variables inter-relate.

\section{Differences in the variables of interest between the clinical group and controls}

The results indicated that in line with previous research (Caglar-Nazali et al., 2014), the clinical ED group reported greater attachment anxiety and attachment avoidance than the controls. In terms of interpersonal RS, the results showed that the clinical ED participants reported greater interpersonal RS compared to controls, providing support for Cardi et al.'s (2013) behavioural study, which found that lifetime ED participants displayed an attentional bias to rejection compared to controls. The current findings also indicated that clinical ED participants reported greater appearance-based RS compared to controls, consistent with previous studies that have found positive associations between appearance-based RS and 
disordered eating (Park, 2007). In regards to social rank, the results indicated that, in line with previous research (Cardi et al., 2014; Troop et al., 2003), clinical ED participants perceived themselves to be lower in social rank than controls.

\section{Assessing the interpersonal model}

\section{The proposed model in clinical EDs}

The results indicate that for individuals with clinical EDs, anxious and avoidant attachment styles were related to disordered eating behaviour through different pathways. Appearancebased RS was a significant mediator of the relationship between anxious attachment and drive for thinness, and between anxious attachment and body dissatisfaction. Avoidant attachment was directly related to body dissatisfaction, and low social rank was a significant mediator of the relationship between avoidant attachment and drive for thinness. The current findings may be interpreted from an attachment perspective. As anxiously attached individuals are preoccupied with avoiding rejection in order to maintain closeness in relationships, they tend to be vigilant to rejection cues (Bartholomew \& Horowitz, 1991). The extent to which RS affects psychological wellbeing depends on whether an individual has experienced rejection in a domain that they value (Ayduk, Downey, \& Kim, 2001), and for clinical ED participants with anxious attachment, physical appearance appears to be a domain of value. Hence, appearance-based RS may trigger disordered eating behaviours such as drive for thinness or body dissatisfaction. These results offer support for the notion that appearance is particularly salient in clinical ED pathology (Rieger et al., 2010). Conversely, individuals with avoidant attachment did not experience the same relationship with appearance-based RS. Instead, 
individuals with avoidant attachment may be dissatisfied with their bodies and this may lead to a drive for thinness through self-perceived low social rank. This may be attributable to the nature of individuals with avoidant attachment, who tend to steer away from close involvement with others to protect themselves against anticipated rejection (Bartholomew \& Horowitz, 1991). It could therefore be argued that because they do not want to get involved in intimate relationships, they are less concerned about rejection based on appearance. Rather, avoidant individuals may place their value in a social domain and therefore experience difficulty with their standing in the social rank. This is consistent with attachment literature that suggests that avoidant individuals have a positive self-evaluation, but a negative evaluation of others (Ein-Dor, Mikulincer, \& Shaver, 2011).

\section{The proposed model in controls}

The current results indicate a different pattern of relationships for the control group. Similar to the clinical group, appearance-based RS was a significant mediator of the relationship between anxious attachment and drive for thinness, and between anxious attachment and body dissatisfaction. In addition, for the controls, appearance-based RS was a significant mediator of the relationship between anxious attachment and bulimia, and avoidant attachment was directly related to bulimia. Further, unlike the clinical group, there were also significant paths involving interpersonal RS. Interpersonal RS was a mediator of the relationship between anxious attachment and body dissatisfaction, as well as between avoidant attachment and body dissatisfaction, where insecure attachment led to interpersonal RS which in turn led to low social rank and subsequent disordered eating behaviour. Social rank was also implicated 
in different pathways for the control group, including in the relationship between anxious attachment and body dissatisfaction, and between avoidant attachment and body dissatisfaction. This is consistent with previous research in community samples (Troop \& Baker, 2008), which found social rank to be predictive of disordered eating.

\section{Limitations}

The present findings should be considered in light of a number of study limitations. Firstly, as the study was cross-sectional, the present findings do not establish causality. Future research should consider the use of longitudinal samples to further examine the mechanisms leading to disordered eating, including mea sur ement of at $\mathrm{t}$ a chment ear 1 ier in 1 ife. Secondly, there are limitations within the literature in the conceptualisation and measurement of social rank. The terminology in the social $\mathrm{rank}$ fiel d draws from social comparison theory, using terms such as "unfavourable social comparison" to describe the perception that one is lower in social st at us than others. However, contrary to traditional social comparison resear ch, social $\mathrm{r}$ ank does not dist inguish between the direction (upward, downward or lateral), or the frequency of the social comparison. There are two previous studies (Bamford \& Halliwell, 2009; Ty \& Francis, 2013) that have found that frequency of social comparison mediates the relationship between insecure attachment and disordered eating. Future research may further develop the current model by incorporating frequency of social comparisons, as this may act as a mechanism through which low perceived social rank leads to disordered eating behaviour. Finally, there are limitations pertaining to the clinical 
ED sample. The recruitment method allowed for the assessment of participants at different stages of their illness/recovery, and with $33 \%$ of the clinical group identifying themselves as recovered, this may have dampened effects in these analyses. Moreover, there may be issues with self-report data such as impression management, especially for clinical ED participants who may have been in treatment at the time. Further, the nature of the sample being predominantly AN-R may have influenced the results of the path analysis for the disordered eating variable of bulimia. This was due to recruitment from tertiary facilities where the majority of patients had AN-R diagnosis and were admitted due to their low weight and associated medical instability (Hay et al., 2014). Future studies may consider the use of a more balanced ED sample with equal distribution of participants across all ED subtypes.

\section{Clinical implications}

The current study builds upon existing knowledge of the role of attachment in the development and maintenance of EDs. Given that high attachment anxiety has been found to be related to greater ED severity and poorer treatment outcomes across all ED subtypes (Illing et al., 2010), while reductions in attachment anxiety and avoidance have been associated with decreases in interpersonal problems (Maxwell, Tasca, Ritchie, Balfour, \& Bissada, 2014), the current study highlights the need for early attachment interventions such as parenting programs and family therapy to improve attachment functioning in order to produce better outcomes for EDs. The current study also expands what is known about negative evaluation in EDs, particularly the maladaptive processing associated with RS and low social rank. These findings may inform existing therapies such as IPT-ED (Rieger et al., 2010) and 
cognitive behaviour therapy for EDs (CBT-E; Fairburn, 2008) by emphasising the roles of RS and social rank in the pathway to disordered eating behaviour and by challenging negative automatic thoughts associated with rejection and social rank comparisons. Behavioural interventions aimed at correcting cognitive bias towards negative social cues (Cardi et al., 2015) have also shown some promise in EDs. Finally, the results indicate the need for clinical interventions to target self-esteem (Sloman, 2008), by expanding the individual's domains of value. In sum, future psychological interventions may therefore not only address underlying insecure attachment but also focus on the cognitive biases that trigger and maintain EDs.

\section{Conclusions}

The current study sought to test a new interpersonal model for eating pathology whereby interpersonal RS, appearance-based RS, and social rank were hypothesised to mediate the relationship between attachment and disordered eating. The results indicate that the relationships within the model differed across clinical ED participants and controls. For the ED group, appearance-based RS and social rank were significant mediators of the relationship between insecure attachment and disordered eating; while for the control group, the relationship between insecure attachment and disordered eating was mediated through multiple pathways involving interpersonal RS, appearance-based RS and social rank. Future research should continue to explore the constructs of RS and social rank, in order to better understand the development of interpersonal difficulties in EDs, and to inform treatments targeting interpersonal functioning for those presenting with disordered eating. 


\section{References}

Allan, S., \& Gilbert, P. (1995). A social comparison scale: Psychometric properties and relationship to psychopathology. Personality and Individual Differences 19, 293-299. doi:10.1016/0191-8869(95)00086-L

Arcelus, J., Haslam, M., Farrow, C., \& Meyer, C. (2013). The role of interpersonal functioning in the maintenance of eating psychopathology: A systematic review and testable model. Clinical Psychology Review, 33(1), 156-167.

doi:10.1016/j.cpr.2012.10.009

Ayduk, O., Downey, G., \& Kim, M. (2001). Rejection sensitivity and depressive symptoms in women. Personality and Social Psychology Bulletin, 27(7), 868-877. doi:10.1177/0146167201277009

Bamford, B., \& Halliwell, E. (2009). Investigating the role of attachment in social comparison theories of eating disorders within a non-clinical female population. European Eating Disorders Review, 17(5), 371-379. doi:10.1002/erv.951

Bartholomew, K., \& Horowitz, L. M. (1991). Attachment styles among young adults: A test of a four-category model. Journal of Personality and Social Psychology, 61(2), 226244. doi:10.1207/S15327957PSPR0602_03

Bowlby, J. (1973). Attachment and loss: Vol II, Separation: Anxiety and anger. New York:

Basic Books.

Caglar-Nazali, H. P., Corfield, F., Cardi, V., Ambwani, S., Leppanen, J., Olabintan, O., ... \& Treasure, J. (2014). A systematic review and meta-analysis of 'Systems for Social 
Processes' in eating disorders. Neuroscience \& Biobehavioral Reviews, 42, 55-92. doi:10.1016/j.neubiorev.2013.12.002.

Calogero, R. M., Park, L. E., Rahemtulla, Z. K., \& Williams, K. C. D. (2010). Predicting excessive body image concerns among British university students: The unique role of appearance-based rejection sensitivity. Body Image, 7(1), 78-81.

doi:10.1016/j.bodyim.2009.09.005

Cardi, V., Di Matteo, R., Corfield, F., \& Treasure, J. (2013). Social reward and rejection sensitivity in eating disorders: An investigation of attentional bias and early experiences. World Journal of Biological Psychiatry, 14(8), 622-633. doi: $10.3109 / 15622975.2012 .665479$.

Cardi, V., Di Matteo, R., Gilbert, P., \& Treasure, J. (2014). Rank perception and selfevaluation in eating disorders. International Journal of Eating Disorders, 47(5), 543552. doi:10.1002/eat.22261.

Cardi, V., Esposito, M., Bird, G., Rhind, C., Yiend, J., Schifano, S., ... Treasure, J. (2015). A preliminary investigation of a novel training to target cognitive biases towards negative social stimuli in Anorexia Nervosa. Journal of Affective Disorders, 188, 188193. doi:10.1016/j.jad.2015.08.019

Cheung, G. W., \& Rensvold, R .B. (2002). Evaluating goodness-of-fit indexes for testing measurement invariance. Structural Equation Modeling, 9(2), 233-255. doi:10.1207/S15328007SEM0902_5

Cohen, J. (1992). A power primer. Psychological bulletin, 112(1), 155-159. doi:10.1037/0033-2909.112.1.155 
Connan, F., Troop, N., Landau, S., Campbell, I. C., \& Treasure, J. (2007). Poor social comparison and the tendency to submissive behavior in anorexia nervosa. International Journal of Eating Disorders, 40(8), 733-739. doi:10.1002/eat.20427

Dakanalis, A., Timko, C. A., Zanetti, M. A., Rinaldi, L., Prunas, A., Carrà, G., ... Clerici, M. (2014). Attachment insecurities, maladaptive perfectionism, and eating disorder symptoms: A latent mediated and moderated structural equation modeling analysis across diagnostic groups. Psychiatry Research, 215(1), 176-184. doi:10.1016/j.psychres.2013.10.039

Downey, G., \& Feldman, S. I. (1996). Implications of rejection sensitivity for intimate relationships. Journal of Personality and Social Psychology, 70(6), 1327-1343. doi:10.1037/0022-3514.70.6.1327

Ein-Dor, T., Mikulincer, M., \& Shaver, P. R. (2011). Attachment insecurities and the processing of threat-related information: Studying the schemas involved in insecure people's coping strategies. Journal of Personality and Social Psychology, 101(1), 7893. doi:10.1037/a0022503

Fairburn, C. G. (2008). Cognitive behavior therapy and eating disorders. New York: Guilford Press.

Fairburn, C. G., \& Beglin, S. J. (1994). Assessment of eating disorders: Interview or selfreport questionnaire? International Journal of Eating Disorders, 16(4), 363-370. doi: 10.1002/1098-108X(199412)16:4<363::AID-EAT2260160405>3.0.CO;2-\# 
Fraley, R. C., \& Shaver, P. R. (2000). Adult romantic attachment: Theoretical developments, emerging controversies, and unanswered questions. Review of General Psychology, 4(2), 132-154. doi:10.1037//1089-2680.4.2.132

Fraley, R. C., Waller, N. G., \& Brennan, K. A. (2000). An item-response theory analysis of self-report measures of adult attachment. Journal of Personality and Social Psychology, 78(2), 350-365. doi:10.1037//0022-3514.78.2.350

Garner, D. M. (2004). Eating Disorder Inventory-3 (EDI-3). Professional Manual. Odessa, FL: Psychological Assessment Resources.

Gilbert, P. (1992). Depression: The Evolution of Powerlessness. Guilford: New York.

Goddard, E., Macdonald, P., Sepulveda, A. R., Naumann, U., Landau, S., Schmidt, U., \& Treasure, J. (2011). Cognitive interpersonal maintenance model of eating disorders: intervention for carers. The British Journal of Psychiatry, 199(3), 225-231.

doi:10.1192/bjp.bp.110.088401

Goddard, E., Salerno, L., Hibbs, R., Raenker, S., Naumann, U., Arcelus, J., ... Lacey, H. (2013). Empirical examination of the interpersonal maintenance model of anorexia nervosa. International Journal of Eating Disorders, 46(8), 867-874. doi:10.1002/eat.22172

Hay, P., Chinn, D., Forbes, D., Madden, S., Newton, R., Sugenor, L. ... Ward, W. (2014) Royal Australian and New Zealand College of Psychiatrists clinical practice guidelines for the treatment of eating disorders. Australian \& New Zealand Journal of Psychiatry, 48(11): 977-1008. doi:10.1177/0004867414555814.

This article is protected by copyright. All rights reserved. 
Hazan, C., \& Shaver, E. (1987). Conceptualizing romantic love as an attachment process. Journal of Personality and Social Psychology, 52(3), 511-524. doi:10.1037/00223514.52.3.511

Illing, V., Tasca, G. A., Balfour, L., \& Bissada, H. (2010). Attachment insecurity predicts eating disorder symptoms and treatment outcomes in a clinical sample of women. The Journal of Nervous and Mental Disease, 198(9), 653-659. doi:10.1097/NMD.0b013e3181ef34b2.

Main, M., Kaplan, N., \& Cassidy, J. (1985). Security in infancy, childhood, and adulthood: A move to the level of representation. Monographs of the Society for Research in Child Development, 50, 66-104. doi:10.2307/3333827

Maxwell, H., Tasca, G. A., Ritchie, K., Balfour, L., \& Bissada, H. (2014). Change in attachment insecurity is related to improved outcomes 1-year post group therapy in women with binge eating disorder. Psychotherapy, 51(1), 57-65.

doi:10.1037/a0031100

Park, L. E. (2007). Appearance-based rejection sensitivity: Implications for mental and physical health, affect, and motivation. Personality and Social Psychology Bulletin, 33(4), 490-504. doi: 10.1177/0146167206296301

Pinto-Gouveia, J., Ferreira, C., \& Duarte, C. (2014). Thinness in the pursuit for social safeness: An integrative model of social rank mentality to explain eating psychopathology. Clinical Psychology \& Psychotherapy, 21(2), 154-165. doi: $10.1002 /$ cpp. 1820

This article is protected by copyright. All rights reserved. 
Rieger, E., Van Buren, D. J., Bishop, M., Tanofsky-Kraff, M., Welch, R., \& Wilfley, D. E. (2010). An eating disorder-specific model of interpersonal psychotherapy (IPT-ED): Causal pathways and treatment implications. Clinical Psychology Review, 30(4), 400410. doi:10.1016/j.cpr.2010.02.001

Schermelleh-Engel, K., Moosbrugger, H., \& Muller, H. (2003). Evaluating the fit of structural equation models: Tests of significance and descriptive goodness-of-fit measures. Methods of Psychological Research Online, 8(8), 23-74.

Schmidt, U., \& Treasure, J. (2006) Anorexia nervosa: Valued and visible. A cognitiveinterpersonalmaintenance model and its implications for research and practice. British Journal of Clinical Psychology, 45(3), 343-366. doi:10.1348/014466505X53902

Shrout, P. E., \& Bolger, N. (2002). Mediation in experimental and nonexperimental studies: New procedures and recommendations. Psychological Methods, 7(4), 422-445.

Sloman, L. (2008). A new comprehensive evolutionary model of depression and anxiety. Journal of Affective Disorders, 106(3), 219-228. doi:10.1016/j.jad.2007.07.008 Tabachnick, B. G., \& Fidell, L. S. (2007). Using Multivariate Statistics (5th ed.). Boston: Allyn \& Bacon.

Treasure, J., \& Schmidt, U. (2013). The cognitive-interpersonal maintenance model of anorexia nervosa revisited: A summary of the evidence for cognitive, socio-emotional and interpersonal predisposing and perpetuating factors. Journal of Eating Disorders, I(1), 13-23. doi:10.1186/2050-2974-1-13

Troop, N. A. (2016). Social rank, rank-related life events and eating pathology. European Eating Disorders Review, 24(1), 75-77. doi:10.1002/erv.2386 
Troop, N. A., Allan, S., Treasure, J. L., \& Katzman, N. (2003). Social comparison and submissive behavior in eating disordered patients. Psychology and Psychotherapy: Theory, Research and Practice, 76(3), 237-249. doi:10.1348/147608303322362479

Troop, N. A., \& Baker, A. H. (2008). The specificity of social rank in eating disorder versus depressive symptoms. Eating Disorders, 16(4), 331-341. doi:10.1080/10640260802115993

Ty, M. \& Francis, A. J. P. (2013). Insecure attachment and disordered eating in women: The mediating processes of social comparison and emotion dysregulation. Eating Disorders: The Journal of Treatment \& Prevention, 21(2), 154-174. doi:10.1080/10640266.2013.761089.

Via, E., Soriano-Mas, C., Sánchez, I., Forcano, L., Harrison, B. J., Davey, C. G., ... Cardoner, N. (2015). Abnormal social reward responses in anorexia nervosa: An fMRI study. PloS One, 10(7), e0133539. doi:10.1371/journal.pone.0133539

Vrtička, P., \& Vuilleumier, P. (2012). Neuroscience of human social interactions and adult attachment style. Frontiers in Human Neuroscience, 6, 212. doi:10.3389/fnhum.2012.00212.

Ward, A., Ramsay, R., \& Treasure, J. (2000). Attachment research in eating disorders. British Journal of Medical Psychology, 73(1), 35-51. doi:10.1348/000711200160282

This article is protected by copyright. All rights reserved. 


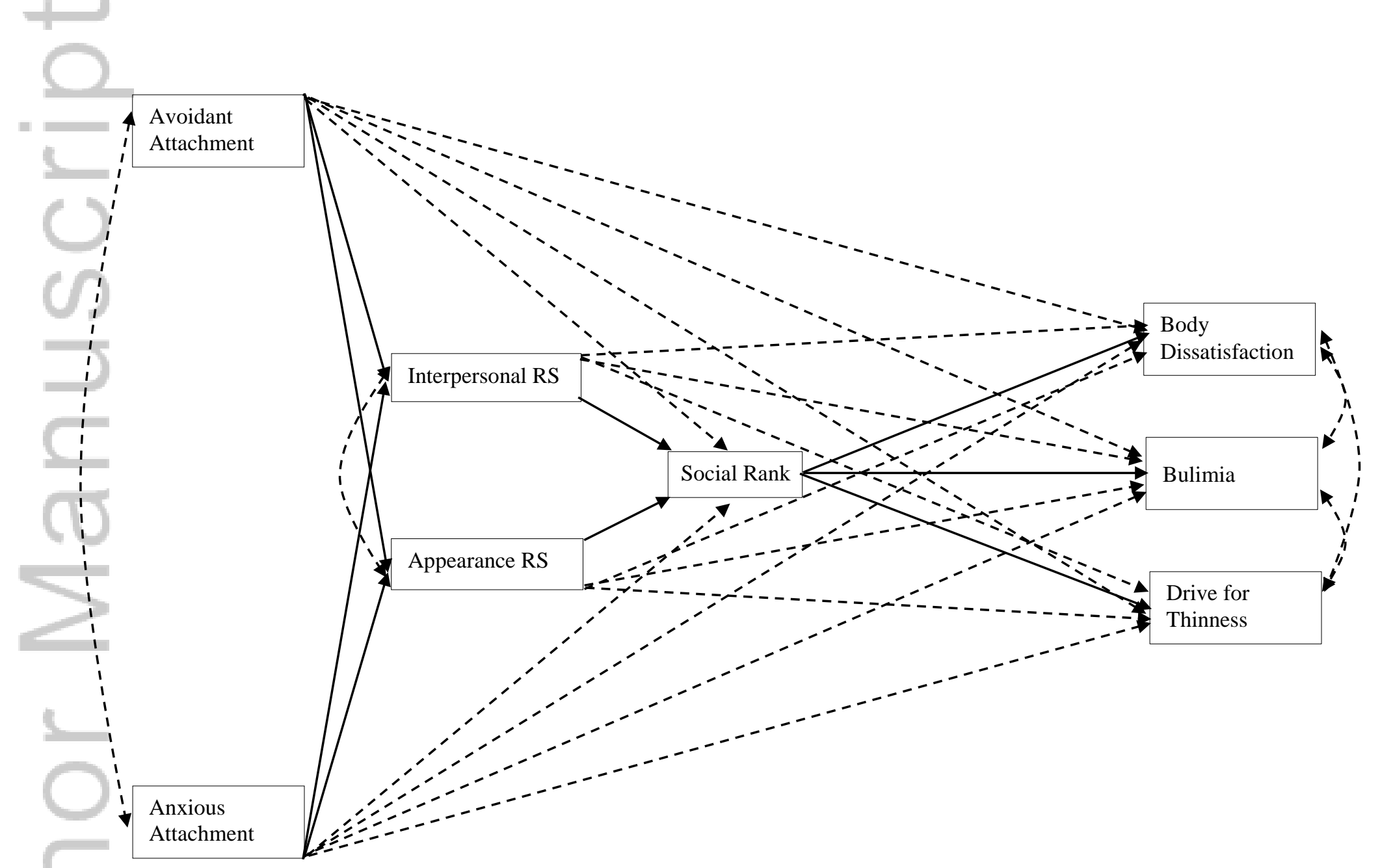

This article is protected by copyright. All rights reserved. 
Figure 1. All potential direct and indirect paths for the hypothesised model. Solid lines represent the tested path model. Dashed lines represent alternative paths to the outcome variables.

This article is protected by copyright. All rights reserved. 
Table 1. Sociodemographic details of study participants.

\begin{tabular}{|c|c|c|c|c|c|c|}
\hline & $\begin{array}{c}\text { Total } \\
(\mathrm{n}=744)\end{array}$ & $\begin{array}{c}\text { EDs } \\
(\mathbf{n}=122)\end{array}$ & $\begin{array}{c}\text { Controls } \\
(n=622)\end{array}$ & $p$ & $\chi^{2}$ & $\begin{array}{c}\text { Cramer's } \\
\text { V }\end{array}$ \\
\hline \multicolumn{7}{|l|}{ Mean (SD) } \\
\hline Age (years) & $22.53(8.55)$ & $25.16(7.60)$ & $22.01(8.63)$ & $<.001$ & & \\
\hline BMI & $22.14(4.71)$ & $20.98(4.59)$ & $22.35(4.70)$ & .005 & & \\
\hline \multicolumn{7}{|l|}{$\mathbf{N}(\%)$} \\
\hline Ethnicity & & & & $<.001$ & 87.672 & .343 \\
\hline Caucasian & $333(44.80)$ & $98(80.30)$ & $235(37.80)$ & & & \\
\hline Aboriginal/Torres Strait & $1(.10)$ & 0 & $1(.20)$ & & & \\
\hline Asian & $264(35.50)$ & $5(4.10)$ & $259(41.60)$ & & & \\
\hline European & $79(10.60)$ & $12(9.80)$ & $67(10.80)$ & & & \\
\hline Middle-Eastern & $10(1.30)$ & 0 & $10(1.60)$ & & & \\
\hline African & $8(1.10)$ & 0 & $8(1.30)$ & & & \\
\hline Hispanic & $2(.30)$ & $1(.80)$ & $1(.20)$ & & & \\
\hline Other & $47(6.30)$ & $6(4.90)$ & $41(6.60)$ & & & \\
\hline $\begin{array}{l}\text { Highest completed } \\
\text { education }\end{array}$ & & & & .049 & 7.864 & .103 \\
\hline Primary & $2(.30)$ & $1(.80)$ & $1(.20)$ & & & \\
\hline Secondary & $370(49.70)$ & $64(52.50)$ & $306(49.20)$ & & & \\
\hline Tertiary & $304(40.90)$ & $40(32.80)$ & $264(42.40)$ & & & \\
\hline Postgraduate & $68(9.10)$ & $17(13.90)$ & $51(8.20)$ & & & \\
\hline Marital status & & & & $<.001$ & 25.185 & .184 \\
\hline Single & $496(66.70)$ & $74(60.70)$ & $422(67.80)$ & & & \\
\hline In a relationship & $190(25.50)$ & $28(23.00)$ & $162(26.00)$ & & & \\
\hline Married & $46(6.20)$ & $15(12.30)$ & $31(5.00)$ & & & \\
\hline Separated & $5(.70)$ & $4(3.28)$ & $1(.20)$ & & & \\
\hline Divorced & $5(.70)$ & $1(.82)$ & $4(.60)$ & & & \\
\hline Widowed & $2(.30)$ & 0 & $2(.30)$ & & & \\
\hline Employment & & & & $<.001$ & 57.307 & .278 \\
\hline Working full-time & $74(9.90)$ & $20(16.40)$ & $54(8.70)$ & & & \\
\hline Working part-time & $180(24.20)$ & $30(24.60)$ & $150(24.10)$ & & & \\
\hline Unemployed & $47(6.30)$ & $24(19.70)$ & $23(3.70)$ & & & \\
\hline Student & $443(59.50)$ & $48(39.30)$ & $395(63.50)$ & & & \\
\hline
\end{tabular}

Note. $p=p$ value (two-tailed) 
Table 2. Descriptive statistics and group difference tests.

\begin{tabular}{cccccc}
\hline & ED $(\mathbf{n = 1 2 2})$ & Controls $(\mathbf{n = 6 2 2})$ & $\boldsymbol{t}$ & $\boldsymbol{p}$ & $\boldsymbol{d}$ \\
\hline Mean (SD) & & & & & \\
Attachment Anxiety & $3.95(1.15)$ & $3.51(1.20)$ & 3.68 & $<.001$ & .37 \\
Attachment Avoidance & $4.03(1.26)$ & $3.28(1.10)$ & 6.74 & $<.001$ & .63 \\
Interpersonal RS & $13.94(6.33)$ & $9.78(3.72)$ & 7.00 & $<.001$ & .80 \\
Appearance-RS & $21.08(9.34)$ & $14.13(7.40)$ & 7.76 & $<.001$ & .82 \\
Social Rank & $40.13(23.10)$ & $63.74(17.39)$ & -10.71 & $<.001$ & 1.15 \\
Drive for Thinness & $17.92(8.24)$ & $8.61(7.15)$ & 12.81 & $<.001$ & 1.21 \\
Body Dissatisfaction & $27.61(10.61)$ & $15.76(9.54)$ & 12.31 & $<.001$ & 1.17 \\
Bulimia & $11.16(9.36)$ & $5.45(5.89)$ & 6.49 & $<.001$ & .79 \\
\hline
\end{tabular}

Note. $p=p$ value (two-tailed), $d=$ Cohen's $d$ 


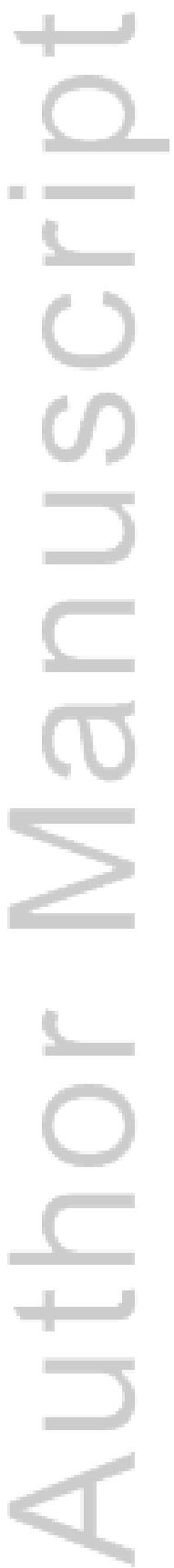

This article is protected by copyright. All rights reserved. 
Table 3. Bivariate correlations between insecure attachment, RS, appearance-based RS, social rank, and disordered eating variables.

\begin{tabular}{|c|c|c|c|c|c|c|c|c|}
\hline 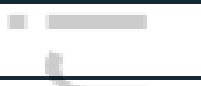 & AttAnx & AttAvoid & $\mathrm{RS}$ & ARS & SR & EDI_DT & EDI_BD & EDI_B \\
\hline AttAnx & 1 & $.475^{* *}$ & $.591^{* *}$ & $.521^{* * *}$ & $-.480^{* *}$ & $.322^{* *}$ & $.426^{* *}$ & $.275^{* *}$ \\
\hline AttAvoid & $.341^{* *}$ & 1 & $.402^{* *}$ & $.282^{* *}$ & $-.491^{* *}$ & $.301^{* *}$ & $.437^{* * *}$ & $.288^{* *}$ \\
\hline $\mathrm{RS}$ & $.470^{* *}$ & $.318^{* *}$ & 1 & $.734^{* *}$ & $-.662^{* *}$ & $.521^{* *}$ & $.583^{* *}$ & $.269^{* *}$ \\
\hline ARS & $.471^{* *}$ & $.200^{* * *}$ & $.519^{* *}$ & 1 & $-.572^{* *}$ & $.610^{* *}$ & $.687^{* *}$ & $.327^{* *}$ \\
\hline SR & $-.446^{* * *}$ & $-.303^{* * *}$ & $-.474^{* *}$ & $-.484^{* *}$ & 1 & $-.529^{* * *}$ & $-.586^{* *}$ & $-.267^{* *}$ \\
\hline EDI_DT & $.277^{* *}$ & $.151^{* *}$ & $.314^{* *}$ & $.545^{* *}$ & $-.239^{* * *}$ & 1 & $.755^{* *}$ & $.472^{* *}$ \\
\hline EDI_BD & $.346^{* *}$ & $.235^{* *}$ & $.370^{* *}$ & $.559^{* *}$ & $-.443^{\text {** }}$ & $.709^{* *}$ & 1 & $.464^{* *}$ \\
\hline EDI_B & $.306^{* *}$ & $.229^{* *}$ & $.333^{* *}$ & $.455^{* *}$ & $-.275^{* *}$ & $.582^{* *}$ & $.554^{* *}$ & 1 \\
\hline
\end{tabular}

**. Correlation is significant at the 0.01 level (2-tailed).

Note. Correlations for the ED sample appear above the diagonal, and correlations for the control sample appear below the diagonal. AttAnx = attachment anxiety; AttAvoid = attachment avoidance; RS = interpersonal RS; ARS = appearance-based RS; SR = social rank; EDI_DT $=$ EDI drive for thinness subscale; EDI_BD = EDI body dissatisfaction subscale; EDI_B = EDI bulimia subscale.

This article is protected by copyright. All rights reserved. 


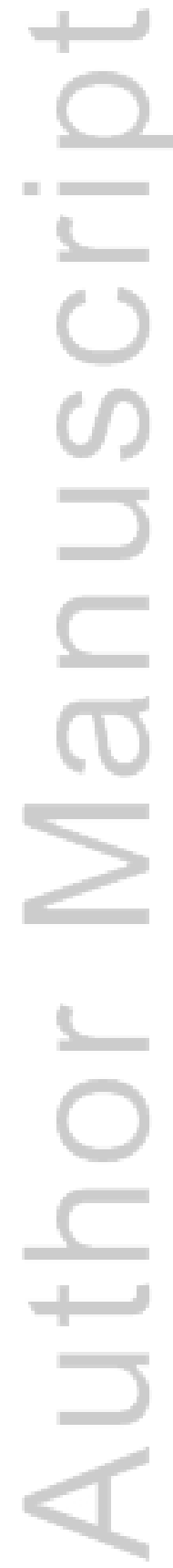

This article is protected by copyright. All rights reserved. 
Table 4. Unstandardised coefficients from analyses testing direct effect pathways between all variables for the clinical ED and control groups.

\begin{tabular}{|c|c|c|c|c|c|c|}
\hline \multirow[b]{2}{*}{$\begin{array}{c}\text { Direct effect } \\
\text { [independent variable } \rightarrow \\
\text { dependent variable] }\end{array}$} & \multicolumn{3}{|c|}{$\operatorname{ED}(\mathrm{N}=122)$} & \multicolumn{3}{|c|}{ Controls $(\mathrm{N}=622)$} \\
\hline & $\mathrm{b}$ & $S E$ & $\begin{array}{l}p \text { value } \\
\text { (two- } \\
\text { tailed) }\end{array}$ & $\mathrm{b}$ & $S E$ & $\begin{array}{l}p \text { value } \\
\text { (two- } \\
\text { tailed) }\end{array}$ \\
\hline AttAnx $\rightarrow$ RS & 2.855 & 0.524 & $<0.001$ & 1.270 & 0.162 & $<0.001$ \\
\hline AttAnx $\rightarrow$ ARS & 4.069 & 0.687 & $<0.001$ & 2.815 & 0.251 & $<0.001$ \\
\hline AttAnx $\rightarrow$ Social rank & -0.274 & 1.777 & 0.877 & -2.631 & 0.655 & $<0.001$ \\
\hline AttAnx $\rightarrow$ EDI_DT & -0.827 & 0.756 & 0.274 & 0.190 & 0.247 & 0.442 \\
\hline AttAnx $\rightarrow$ EDI_BD & -0.339 & 0.765 & 0.658 & 0.308 & 0.297 & 0.300 \\
\hline AttAnx $\rightarrow$ EDI_B & 0.494 & 1.131 & 0.663 & 0.322 & 0.242 & 0.182 \\
\hline AttAvoid $\rightarrow$ RS & 0.787 & 0.520 & 0.132 & 0.596 & 0.155 & $<0.001$ \\
\hline AttAvoid $\rightarrow$ ARS & 0.334 & 0.684 & 0.625 & 0.290 & 0.276 & 0.293 \\
\hline AttAvoid $\rightarrow$ Social rank & -4.889 & 1.202 & $<0.001$ & -1.854 & 0.615 & 0.003 \\
\hline AttAvoid $\rightarrow$ EDI_DT & 0.405 & 0.525 & 0.440 & 0.180 & 0.242 & 0.456 \\
\hline AttAvoid $\rightarrow$ EDI_BD & 1.535 & 0.542 & 0.005 & 0.564 & 0.325 & 0.083 \\
\hline AttAvoid $\rightarrow$ EDI_B & 0.978 & 0.845 & 0.247 & 0.517 & 0.234 & $\mathbf{0 . 0 2 7}$ \\
\hline $\mathrm{RS} \rightarrow$ Social rank & -1.476 & 0.331 & $<0.001$ & -1.003 & 0.202 & $<0.001$ \\
\hline RS $\rightarrow$ EDI_DT & 0.069 & 0.177 & 0.696 & 0.089 & 0.081 & 0.274 \\
\hline $\mathrm{RS} \rightarrow$ EDI_BD & 0.010 & 0.146 & 0.948 & 0.054 & 0.110 & 0.622 \\
\hline $\mathrm{RS} \rightarrow$ EDI_B & -0.072 & 0.247 & 0.772 & 0.139 & 0.088 & 0.114 \\
\hline ARS $\rightarrow$ Social rank & -0.477 & 0.224 & 0.033 & -0.617 & 0.103 & $<0.001$ \\
\hline ARS $\rightarrow$ EDI_DT & 0.412 & 0.092 & $<0.001$ & 0.500 & 0.040 & $<0.001$ \\
\hline ARS $\rightarrow$ EDI_BD & 0.617 & 0.116 & $<0.001$ & 0.524 & 0.053 & $<0.001$ \\
\hline ARS $\rightarrow$ EDI_B & 0.265 & 0.133 & 0.047 & 0.281 & 0.041 & $<0.001$ \\
\hline Social rank $\rightarrow$ EDI_DT & -0.086 & 0.036 & 0.015 & 0.029 & 0.019 & 0.117 \\
\hline Social rank $\rightarrow$ EDI_BD & -0.090 & 0.044 & 0.043 & -0.105 & 0.024 & $<0.001$ \\
\hline Social rank $\rightarrow$ EDI_B & -0.014 & 0.050 & 0.786 & 0.004 & 0.022 & 0.844 \\
\hline
\end{tabular}




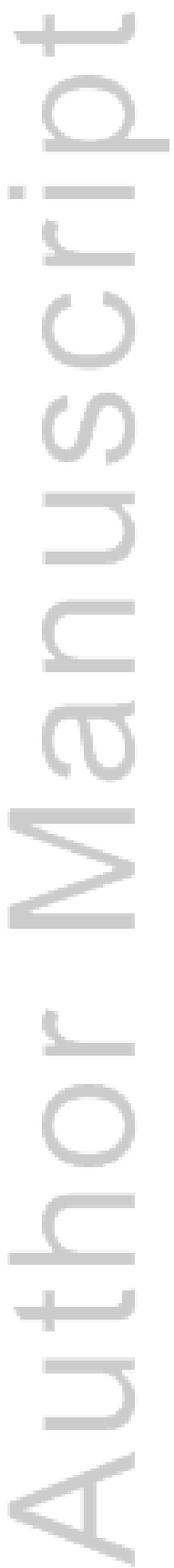

This article is protected by copyright. All rights reserved. 


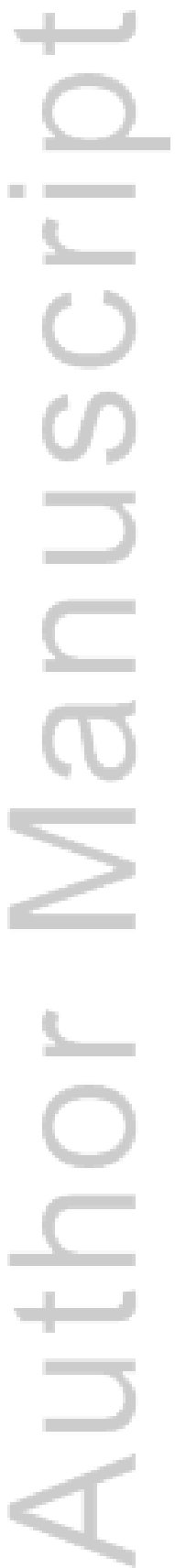

This article is protected by copyright. All rights reserved. 
Table 5. Standardised coefficients from analyses testing indirect effect pathways from insecure

attachment to disordered eating for the clinical ED group and the control group.

\begin{tabular}{|c|c|c|c|c|c|c|}
\hline \multirow[b]{2}{*}{$\begin{array}{c}\text { Indirect effect } \\
\text { [independent variable } \rightarrow \text { mediator variable(s) } \\
\rightarrow \text { dependent variable] }\end{array}$} & \multicolumn{3}{|c|}{ ED } & \multicolumn{3}{|c|}{ Controls } \\
\hline & $\beta$ & $S E$ & $\begin{array}{l}p \text { value } \\
\text { (two- } \\
\text { tailed) }\end{array}$ & $\beta$ & $S E$ & $\begin{array}{l}p \text { value } \\
\text { (two- } \\
\text { tailed) }\end{array}$ \\
\hline AttAnx $\rightarrow$ RS $\rightarrow$ EDI_DT & 0.027 & 0.070 & 0.697 & 0.019 & 0.018 & 0.283 \\
\hline AttAnx $\rightarrow$ ARS $\rightarrow$ EDI_DT & 0.233 & 0.064 & $<0.001$ & 0.239 & 0.028 & $<0.001$ \\
\hline AttAnx $\rightarrow$ Social rank $\rightarrow$ EDI_DT & 0.003 & 0.023 & 0.889 & -0.013 & 0.009 & 0.146 \\
\hline AttAnx $\rightarrow$ RS $\rightarrow$ Social rank $\bar{\rightarrow}$ EDI_DT & 0.050 & 0.027 & 0.062 & -0.006 & 0.004 & 0.160 \\
\hline AttAnx $\rightarrow$ ARS $\rightarrow$ Social rank $\rightarrow$ EDI_DT & 0.023 & 0.016 & 0.139 & -0.009 & 0.006 & 0.140 \\
\hline AttAnx $\rightarrow$ RS $\rightarrow$ EDI_BD & 0.003 & 0.046 & 0.949 & 0.009 & 0.018 & 0.624 \\
\hline AttAnx $\rightarrow$ ARS $\rightarrow$ EDI_BD & 0.271 & 0.072 & $<0.001$ & 0.187 & 0.025 & $<0.001$ \\
\hline AttAnx $\rightarrow$ Social rank $\bar{\rightarrow}$ EDI_BD & 0.003 & 0.019 & 0.891 & 0.035 & 0.012 & 0.004 \\
\hline AttAnx $\rightarrow$ RS $\rightarrow$ Social rank $\rightarrow$ EDI_BD & 0.041 & 0.024 & 0.088 & 0.017 & 0.006 & 0.003 \\
\hline AttAnx $\rightarrow$ ARS $\rightarrow$ Social rank $\rightarrow$ EDI_BD & 0.019 & 0.013 & 0.158 & 0.023 & 0.006 & $<0.001$ \\
\hline AttAnx $\rightarrow$ RS $\rightarrow$ EDI_B & -0.025 & 0.088 & 0.777 & 0.036 & 0.025 & 0.144 \\
\hline AttAnx $\rightarrow$ ARS $\rightarrow$ EDI_B & 0.132 & 0.069 & 0.055 & 0.162 & 0.025 & $<0.001$ \\
\hline AttAnx $\rightarrow$ Social rank $\rightarrow$ EDI_B & 0.000 & 0.011 & 0.968 & -0.002 & 0.012 & 0.848 \\
\hline AttAnx $\rightarrow$ RS $\rightarrow$ Social rank $\rightarrow$ EDI_B & 0.007 & 0.027 & 0.798 & -0.001 & 0.006 & 0.848 \\
\hline AttAnx $\rightarrow$ ARS $\rightarrow$ Social rank $\rightarrow$ EDI_B & 0.003 & 0.013 & 0.804 & -0.002 & 0.008 & 0.846 \\
\hline AttAvoid $\rightarrow$ RS $\rightarrow$ EDI_DT & 0.008 & 0.025 & 0.743 & 0.008 & 0.008 & 0.308 \\
\hline AttAvoid $\rightarrow$ ARS $\rightarrow$ EDI_DT & 0.021 & 0.044 & 0.632 & 0.023 & 0.022 & 0.297 \\
\hline AttAvoid $\rightarrow$ Social rank $\rightarrow$ EDI_DT & 0.064 & 0.032 & 0.042 & -0.008 & 0.006 & 0.179 \\
\hline AttAvoid $\rightarrow$ RS $\rightarrow$ Social rank $\rightarrow$ EDI_DT & 0.015 & 0.013 & 0.230 & -0.003 & 0.002 & 0.184 \\
\hline AttAvoid $\rightarrow$ ARS $\rightarrow$ Social rank $\rightarrow$ EDI_DT & 0.002 & 0.005 & 0.681 & -0.001 & 0.001 & 0.428 \\
\hline AttAvoid $\rightarrow$ RS $\rightarrow$ EDI_BD & 0.001 & 0.016 & 0.956 & 0.004 & 0.008 & 0.635 \\
\hline AttAvoid $\rightarrow$ ARS $\rightarrow$ EDI_BD & 0.025 & 0.050 & 0.625 & 0.018 & 0.017 & 0.298 \\
\hline AttAvoid $\rightarrow$ Social rank $\bar{\rightarrow}$ EDI_BD & 0.052 & 0.029 & 0.072 & 0.023 & 0.009 & 0.016 \\
\hline AttAvoid $\rightarrow$ RS $\rightarrow$ Social rank $\rightarrow$ EDI_BD & 0.012 & 0.012 & 0.291 & 0.007 & 0.003 & 0.015 \\
\hline AttAvoid $\rightarrow$ ARS $\rightarrow$ Social rank $\rightarrow$ EDI_BD & 0.002 & 0.004 & 0.696 & 0.002 & 0.002 & 0.339 \\
\hline AttAvoid $\rightarrow$ RS $\rightarrow$ EDI_B & -0.008 & 0.032 & 0.810 & 0.016 & 0.010 & 0.121 \\
\hline AttAvoid $\rightarrow$ ARS $\rightarrow$ EDI_B & 0.012 & 0.027 & 0.658 & 0.015 & 0.015 & 0.312 \\
\hline AttAvoid $\rightarrow$ Social rank $\rightarrow$ EDI_B & 0.009 & 0.034 & 0.794 & -0.001 & 0.008 & 0.852 \\
\hline AttAvoid $\rightarrow$ RS $\rightarrow$ Social rank $\rightarrow$ EDI_B & 0.002 & 0.010 & 0.825 & 0.000 & 0.003 & 0.853 \\
\hline AttAvoid $\rightarrow$ ARS $\rightarrow$ Social rank $\rightarrow$ EDI_B & 0.000 & 0.003 & 0.914 & 0.000 & 0.001 & 0.886 \\
\hline
\end{tabular}

Note. $\beta=$ standardised beta weight, $S E=$ standard error; AttAnx = attachment anxiety; AttAvoid = attachment avoidance; RS = interpersonal RS; ARS = appearance-based RS; EDI_DT = EDI drive for thinness subscale; EDI_BD = EDI body dissatisfaction subscale; EDI_B = EDI bulimia subscale. 


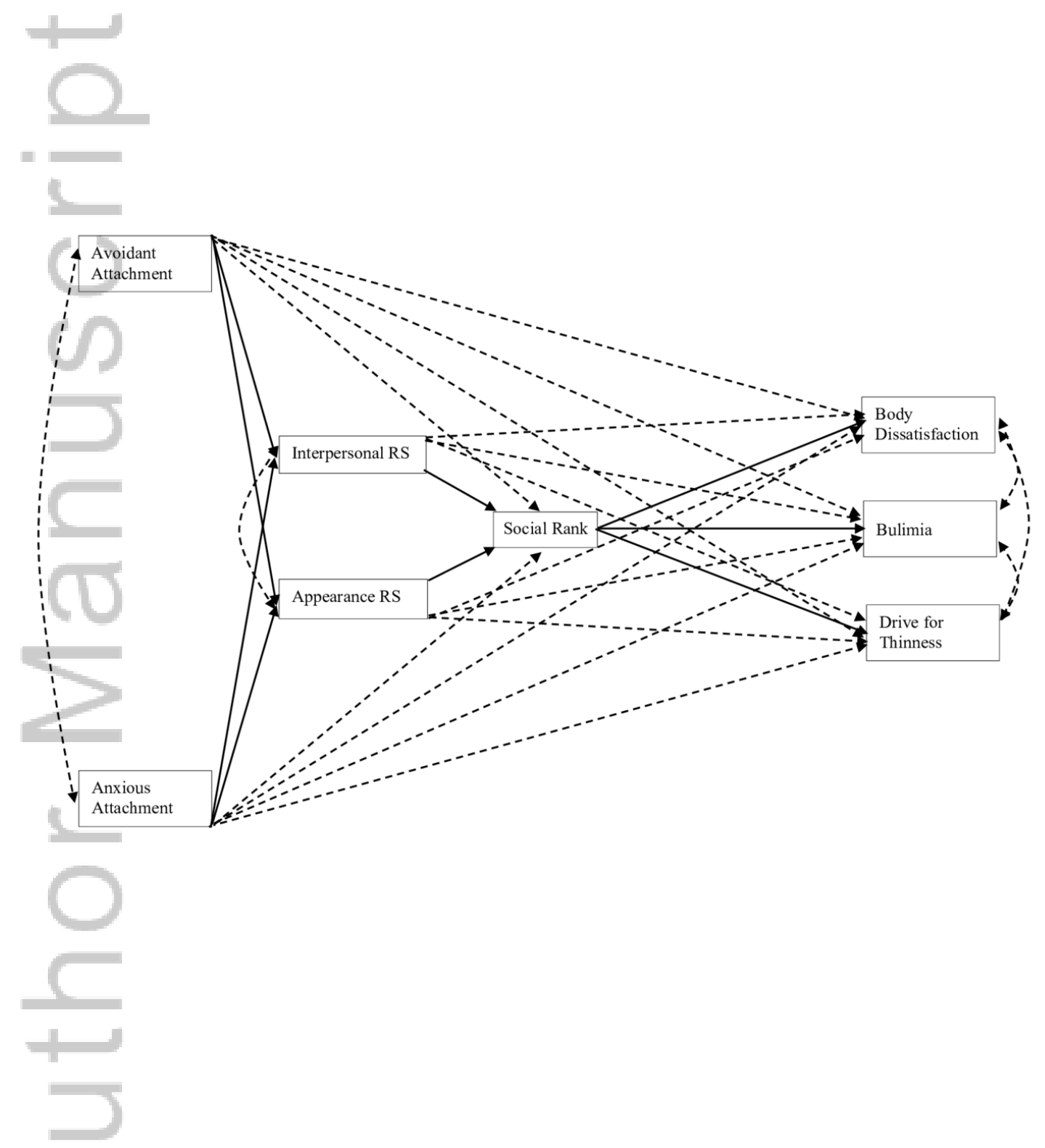

ERV_2537_F1.tiff

This article is protected by copyright. All rights reserved. 


\section{University Library}

\section{- M M N E R VA A gateway to Melbourne's research publications}

Minerva Access is the Institutional Repository of The University of Melbourne

Author/s:

De Paoli, T;Fuller-Tyszkiewicz, M;Halliwell, E;Puccio, F;Krug, I

Title:

Social Rank and Rejection Sensitivity as Mediators of the Relationship between Insecure Attachment and Disordered Eating

Date:

2017-11-01

Citation:

De Paoli, T., Fuller-Tyszkiewicz, M., Halliwell, E., Puccio, F. \& Krug, I. (2017). Social Rank and Rejection Sensitivity as Mediators of the Relationship between Insecure Attachment and Disordered Eating. EUROPEAN EATING DISORDERS REVIEW, 25 (6), pp.469-478. https:// doi.org/10.1002/erv.2537.

Persistent Link:

http://hdl.handle.net/11343/293234 